\title{
Expression of Vascular Endothelial Growth Factor and Its Receptors in Thyroid Nodular Hyperplasia and Papillary Thyroid Carcinoma: A Tertiary Health Care Centre Based Study
}

\author{
Nur Hidayati Mohamad Pakarul Razy, Wan Faiziah Wan Abdul Rahman'*, \\ Thin Thin Win ${ }^{2}$
}

\begin{abstract}
Introduction: Vascular endothelial growth factor (VEGF) is an angiogenic factor that plays an important role in thyroid cancer. VEGF is known to have high affinity to VEGF receptors such as VEGFR-1 (Flt-1) and VEGFR-2 (KDR). Papillary thyroid carcinoma (PTC) is the most common thyroid cancer and studies showed the increasing incidence of PTC arising in nodular hyperplasia. Targeted therapy on these growth factors and receptors are used in management of both differentiated and undifferentiated thyroid carcinoma. This study aims to determine the expression of VEGF and VEGF receptors (VEGFR) in thyroid nodular hyperplasia and PTC. Methods: A cross-sectional study based on paraffinized archival tissue blocks of 113 nodular hyperplasias and 67 PTC from the thyroidectomy specimens in the year of 2003 to 2014. The tissue sections were then stained by immunohistochemistry for VEGF, VEGFR-1 and VEGFR-2. The lymph node involvement and extrathyroid extension also were determined. Results: The mean age of PTC patients was $44.7 \pm 15.8$ years and nodular hyperplasia were $42.2 \pm 13.6$ years. There was a statistical difference of VEGFR-1 $(p=0.028)$ and VEGFR-2 $(p=0.003)$ expression between nodular hyperplasia and PTC. However, no significant difference of VEGF expression $(p=0.576)$ between both diseases. Co-expression of VEGF and VEGFR-1 was significant in both nodular hyperplasia $(p=0.016)$ and PTC $(p=0.03)$, meanwhile no relevant relationship for VEGF and VEGFR-2 expression ( $p>0.05)$. No significant association $(p>0.05)$ between lymph node status and extrathyroid extension with age groups, gender, VEGF and VEGFR expression. Conclusions: VEGF, VEGFR-1 and VEGFR-2 showed overexpression in both nodular hyperplasia and PTC. The expression of VEGFR-1 and VEGFR-2 are more significant in PTC with relevant co-expression of VEGF and VEGFR-1. Therefore, the inhibition of VEGFR offers a promising prospect for tumour management in thyroid carcinoma.
\end{abstract}

Keywords: VEGF- VEGFR-1- VEGFR-2- thyroid nodular hyperplasia- papillary thyroid carcinoma

Asian Pac J Cancer Prev, 20 (1), 277-282

\section{Introduction}

Thyroid cancer, particularly papillary thyroid carcinoma (PTC) is highly associated with multi-nodular goitre pathologically termed nodular hyperplasia $(\mathrm{NH})$ (Sachmechi et al., 2000; Gandolfi et al., 2004). Many cases of PTC were seen developing from pre-existing NH (Othman et al., 2009; Younes et al., 2006).

Many growth factors play role in carcinogenesis of many solid malignant tumours including thyroid cancer. Role of many growth factors and their receptors in follicular derived thyroid cancer have been proved. With that, various therapeutic agents are used in targeting growth factors and their receptors such as vasculoendothelial growth factor receptor (VEGFR), epidermal growth factor receptors (EGFR), platelet drived growth factor (PDGF) and its receptors (PDGFR). (Parameswaran et al., 2010). Among them, vascular endothelial growth factor (VEGF) is an angiogenic factor that plays an essential role in thyroid cancer (Dvorak et al., 1995). VEGF is known to have high affinity to VEGF receptors such as VEGFR-1 (Flt-1) and VEGFR-2 (KDR) (Johnson et al., 2012). The importance of angiogenesis in tumour progression and metastasis is well-recognised and its potential role in prognosis and the therapeutic application has led to many ongoing clinical researches (Younes et al., 2006). VEGF signalling plays a vital role in thyroid carcinogenesis and angiogenesis of thyroid cancer carcinoma (Vieira et al., 2005). VEGF inhibitors have been used to block the angiogenesis and to induce apoptosis 
in thyroid cancer (Peng et al., 2015). Both VEGF and VEGFR have been observed to have prominent prognostic association in differentiated thyroid cancer (Zhou et al., 2012; Abdel-Rahman, 2015). Tyrosine kinase inhibitors which targeted on VEGF and VEGFR have been used in the therapeutic management of differentiated thyroid carcinoma (Fallahi et al., 2013).

East coast of Malaysia particularly Kelantan State has a high incidence of multinodular goitre and thyroid cancer particularly PTC. Thyroid cancer arising from multi-nodular goitre are high in a population living in an iodine-deficiency area (Othman et al., 2009). Most of the previous studies on VEGF and its receptors were done on thyroid carcinoma only. In this study, based on the previous research findings on thyroid lesions in the Kelantan State of Malaysia, we studied the immunohistochemical expression of VEGF and its receptors, VEGFR-1 and VEGFR-2 in both PTC and $\mathrm{NH}$ cases; and examined their association with clinicopathological parameters of PTC in the largest tertiary health care centre of Kelantan State.

\section{Materials and Methods}

\section{Methods}

It is a comparative cross-sectional (retrospective) study which included thyroidectomy specimens of 113 cases of NH and 67 cases of PTC from 2003 to 2014. Lymph node involvement and extra-thyroidal extension were recorded in PTC cases. The retrieved Formalin-fixed paraffin-embedded tissue blocks of all these cases were sectioned into 3-4 $\mu \mathrm{m}$ thickness and placed on Poly-L Lysine slides. After de-paraffinization, antigen retrieval was carried out with citrate buffer at $\mathrm{pH} 6.0$ using a pressure cooker. Polyclonal primary antibodies were applied and incubated for 30 minutes at room temperature. Polyclonal rabbit anti-human VEGF (Santa Cruz), monoclonal rabbit anti-VEGFR-1 (Santa Cruz) and polyclonal rabbit anti-human VEGFR-2 (Santa Cruz) were used as primary antibodies. For a secondary antibody and detection system, HRP polymer solution, a buffered solution containing hydrogen peroxide and a detector agent of Di-amino benzidine (a chromogen in organic solvent) were used.

All slides were analysed under a microscope (Olympus CX31) with $400 \times$ magnifications and scored using a semi-quantitative scoring based on the intensity score and proportion score. The intensity staining was graded on the following scale: (a) 0 (absence of staining); (b) 1 (weak staining); (c) 2 (moderate, light brown in color, not completely filling cytoplasm) and (c) 3 (intense staining of cytoplasm, very dark brown, completely filling the cell). The proportion score is the estimation of the proportion of the positive cells within the tissue on the entire slide. The scoring is divided into following categories: (a) 0 (absence of staining); (b) 1 (less than $30 \%$ of stained thyroid follicular cells); (c) 2 (30 to 60\%); and (d) 3 (more than $60 \%$ ). Sum of multiplication both scores allowed the final quotation ranging from 0 to 9 . The final classification divided into 2 categories: (a) low expression (1 to 5) and (b) high expression (6 to 9) (Klein et al., 2001).

All the results were analysed using the statistical package for in the social sciences (SPSS) software. The chi-square test was used to determine the association and differences in staining between benign and malignant groups with other clinicopathological variables. A p value of $\leq 0.05$ was considered statistically significant.

\section{Results}

In this study, the mean age was $44.7 \pm 15.8$ years for PTC cases and $42.2 \pm 13.6$ years for NH cases. Male: female (\%) were 14: 86 and 22: 78 in NH and PTC respectively. Lymph node involvement in PTC was seen in 15 cases $(22 \%)$ and extra-thyroidal extension was seen in 2 cases (3\%) of PTC.

VEGF immunohistochemical staining in thyroid epithelial cells was detected in all 113 subjects of $\mathrm{NH}$ and 67 cases of PTC, and it was high in most of the cases of NH and PTC. There was no statistical difference $(p=0.576)$ in VEGF expression between NH and PTC. Table 1 summarised the frequency of VEGF, VEGFR-1 and VEGFR-2 expression in both nodular hyperplasia and

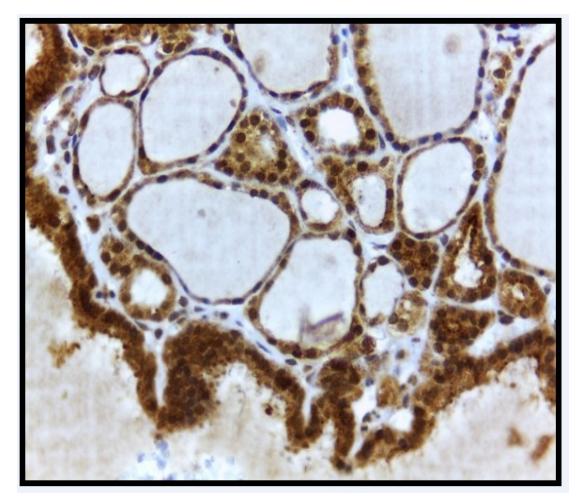

Figure 1. Strong expression of VEGF in cytoplasm of thyrocytes of nodular hyperplasia. Original magnification $\mathrm{x} 400$.

Table 1. Frequency of VEGF, VEGFR-1 and VEGFR-2 Expression in NH and PTC.

\begin{tabular}{|c|c|c|c|c|c|c|c|c|c|c|c|c|c|c|c|c|c|}
\hline \multirow[t]{2}{*}{$\begin{array}{l}\text { VEGF } \\
\text { expression }\end{array}$} & \multicolumn{2}{|c|}{$\begin{array}{c}\text { NH } \\
(n=113)\end{array}$} & \multicolumn{2}{|c|}{$\begin{array}{c}\text { PTC } \\
(n=67)\end{array}$} & \multirow[t]{2}{*}{$\begin{array}{c}\mathrm{p}- \\
\text { value }\end{array}$} & \multirow[t]{2}{*}{$\begin{array}{l}\text { VEGFR-1 } \\
\text { expression }\end{array}$} & \multicolumn{2}{|c|}{$\begin{array}{c}\text { NH } \\
(n=113)\end{array}$} & \multicolumn{2}{|c|}{$\begin{array}{c}\text { PTC } \\
(n=67)\end{array}$} & \multirow[t]{2}{*}{$\begin{array}{c}\mathrm{p}- \\
\text { value }\end{array}$} & \multirow[t]{2}{*}{$\begin{array}{l}\text { VEGFR-2 } \\
\text { expression }\end{array}$} & \multicolumn{2}{|c|}{$\begin{array}{c}\text { NH } \\
(n=113)\end{array}$} & \multicolumn{2}{|c|}{$\begin{array}{c}\text { PTC } \\
(n=67)\end{array}$} & \multirow[t]{2}{*}{$\begin{array}{c}\mathrm{p}- \\
\text { value }\end{array}$} \\
\hline & $\mathrm{n}$ & $\%$ & $\mathrm{n}$ & $\%$ & & & $\mathrm{n}$ & $\%$ & $\mathrm{n}$ & $\%$ & & & $\mathrm{n}$ & $\%$ & $\mathrm{n}$ & $\%$ & \\
\hline Negative & 0 & 0 & 0 & 0 & & Negative & 5 & 4 & 0 & 0 & & Negative & 17 & 15 & 0 & 0 & \\
\hline $\begin{array}{l}\text { Low } \\
\text { (Score 1-4) }\end{array}$ & 15 & 13 & 7 & 10 & 0.576 & $\begin{array}{c}\text { Low } \\
\text { (Score 1-4) }\end{array}$ & 38 & 34 & 14 & 21 & 0.028 & $\begin{array}{c}\text { Low } \\
\text { (Score 1-4) }\end{array}$ & 52 & 46 & 39 & 58 & 0.003 \\
\hline $\begin{array}{l}\text { High } \\
\text { (Score 6-9) }\end{array}$ & 98 & 87 & 60 & 90 & & $\begin{array}{c}\text { High } \\
\text { (Score 6-9) }\end{array}$ & 70 & 62 & 53 & 79 & & $\begin{array}{c}\text { High } \\
\text { (Score 6-9) }\end{array}$ & 44 & 39 & 28 & 42 & \\
\hline
\end{tabular}


DOI:10.31557/APJCP.2019.20.1.277

Vascular Endothelial Growth Factor in Nodular Hyperplasia and Papillary Thyroid Carcinoma

Table 2. The Association in Clinicopathological Data of Patients Diagnosed with PTC with VEGF, VEGFR-1 and VEGFR-2 Expression

\begin{tabular}{|c|c|c|c|c|c|c|c|c|c|}
\hline & \multicolumn{3}{|c|}{ VEGF } & \multicolumn{3}{|c|}{ VEGFR-1 } & \multicolumn{3}{|c|}{ VEGFR-2 } \\
\hline & $\begin{array}{c}\text { Low } \\
\text { n (\%) }\end{array}$ & $\begin{array}{l}\text { High } \\
\text { n (\%) }\end{array}$ & p-value & $\begin{array}{l}\text { Low } \\
\text { n (\%) }\end{array}$ & $\begin{array}{l}\text { High } \\
\text { n (\%) }\end{array}$ & p-value & $\begin{array}{l}\text { Low } \\
\text { n (\%) }\end{array}$ & $\begin{array}{l}\text { High } \\
\text { n (\%) }\end{array}$ & p-value \\
\hline \multicolumn{10}{|c|}{ Lymph node involvement } \\
\hline Positive $(\mathrm{n}=15)$ & $1(7)$ & $14(93)$ & $\mathrm{p}>0.95$ & $2(13)$ & $13(87)$ & $\mathrm{p}=0.178$ & $6(40)$ & $9(60)$ & $\mathrm{p}>0.95$ \\
\hline Negative $(n=4)$ & $0(0)$ & $4(100)$ & & $2(50)$ & $2(50)$ & & $2(50)$ & $2(50)$ & \\
\hline \multicolumn{10}{|c|}{ Extra-thyroidal extension } \\
\hline Positive $(\mathrm{n}=2)$ & $0(0)$ & $2(100)$ & $\mathrm{p}>0.95$ & $1(50)$ & $1(50)$ & $\mathrm{p}=0.352$ & $0(0)$ & $2(100)$ & $\mathrm{p}=0.486$ \\
\hline Negative $(n=19)$ & $1(5)$ & $18(95)$ & & $3(16)$ & $16(84)$ & & $9(47)$ & $10(53)$ & \\
\hline
\end{tabular}

Table 3. The Association of Expression of VEGF, VEGFR-1 and VEGFR-2 in NH ( $\mathrm{n}=113)$

\begin{tabular}{|c|c|c|c|c|c|c|c|}
\hline \multicolumn{2}{|c|}{ Expression } & \multirow[t]{2}{*}{ n $(\%)$} & \multirow[t]{2}{*}{ p-value } & \multicolumn{2}{|c|}{ expression } & \multirow[t]{2}{*}{ n (\%) } & \multirow[t]{2}{*}{ p-value } \\
\hline VEGF & VEGFR-1 & & & VEGF & VEGFR-2 & & \\
\hline Low & Negative & $3(3 \%)$ & 0.016 & Low & Negative & $2(2 \%)$ & $>0.95$ \\
\hline High & Negative & $2(2 \%)$ & & High & Negative & $15(13 \%)$ & \\
\hline Low & Positive & $12(11 \%)$ & & Low & Positive & $13(11.5 \%)$ & \\
\hline High & Positive & $96(85 \%)$ & & High & Positive & $83(74 \%)$ & \\
\hline
\end{tabular}

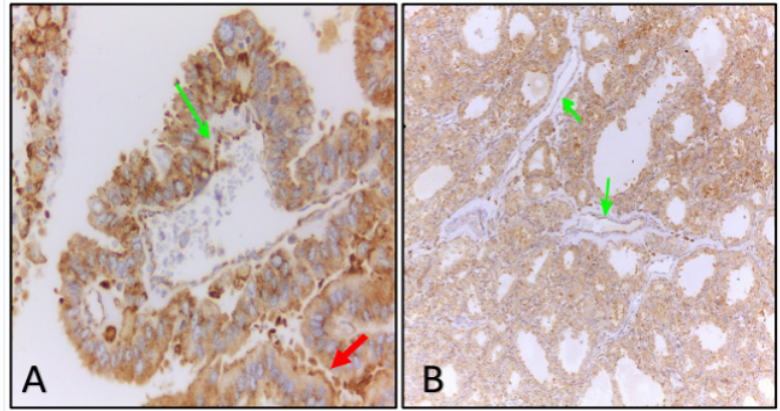

Figure 2. Immunohistochemical Detection of VEGFR-1 in Cytoplasm and Membrane (Red Arrow) of Papillary Thyroid Carcinoma Cells (A) and cytoplasm of thyrocytes of nodular hyperplasia (B). Green arrows indicate VEGFR-1 staining in endothelial lining of blood vessels. Original magnifications, A, x 400 and B, x 100.

papillary thyroid carcinoma. Figure 1 showed a strong expression of VEGF in the cytoplasm of thyroid follicular cells of nodular hyperplasia.

VEGFR-1 was positive in 108 cases (96\%) of NH and all cases of PTC. VEGFR-2 was positive in 98 cases $(85 \%)$ of $\mathrm{NH}$ and all cases of PTC. There was a statistical difference in VEGFR-1 $(\mathrm{p}=0.028)$ and VEGFR-2 $(p=0.003)$ expression between NH and PTC. In PTC cases, there was no association between lymph node status with expression of VEGF ( $p>0.95)$, VEGFR-1 $(p=0.178)$ and VEGFR-2 ( $>0.95)$; and also no significant association

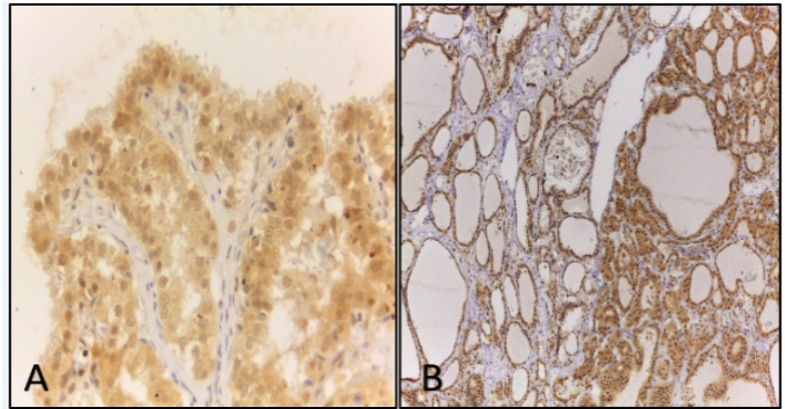

Figure 3. Immunohistochemical Detection of VEGFR-2 in Cytoplasm of Papillary Thyroid Carcinoma Cells (A) and cytoplasm of thyrocytes of nodular hyperplasia (B). Original magnifications, A, x 400 and B, x 100.

found between extra-thyroid extension with expression of VEGF ( $p>0.95)$, VEGFR-1 $(p=0.352)$ and VEGFR-2 $(p=0.486)$. Co-expression of VEGF and VEGFR-1 was significant in both $\mathrm{NH}(\mathrm{p}=0.016)$ and PTC $(\mathrm{p}=0.03)$, meanwhile, there was no significant relationship between VEGF and VEGFR-2 expression.

The association in clinicopathological data of PTC cases with VEGF, VEGFR-1 and VEGFR-2 expression were shown in Table 2. Table 3 and 4 showed the association of expression of VEGF, VEGFR-1 and VEGFR-2 in NH and PTC cases respectively. Immunohistochemical expression of VEGFR-1 and VEGFR-2 in thyroid follicular cells of NH and PTC were shown in Figure 2 and Figure 3.

Table 4. The Association of Expression of VEGF, VEGFR-1 and VEGFR-2 in PTC $(n=67)$

\begin{tabular}{|c|c|c|c|c|c|c|c|}
\hline \multicolumn{2}{|c|}{ Expression } & \multirow[t]{2}{*}{$\mathrm{n}(\%)$} & \multirow[t]{2}{*}{ p-value } & \multicolumn{2}{|c|}{ Expression } & \multirow[t]{2}{*}{$\mathrm{n}(\%)$} & \multirow[t]{2}{*}{ p-value } \\
\hline VEGF & VEGFR-1 & & & VEGF & VEGFR-2 & & \\
\hline Low & Low & $5(8 \%)$ & 0.03 & Low & Low & $6(9 \%)$ & $>0.225$ \\
\hline High & High & $51(76 \%)$ & & High & High & $27(40 \%)$ & \\
\hline Low & High & $2(3 \%)$ & & Low & High & $1(2 \%)$ & \\
\hline High & Low & $9(13 \%)$ & & High & Low & $33(49 \%)$ & \\
\hline
\end{tabular}




\section{Discussion}

Angiogenesis plays an essential role in the growth of both benign thyroid tissue, such as multinodular goitre, Grave disease, and thyroid cancer. Various growth factors VEGF, fibroblast growth factors, placental derived growth factors and many more produced by the cancer cells stimulate tumour angiogenesis via paracrine action. Among them, VEGF is the most important and most powerful growth factor for thyroid cancer development and metastasis.

This study analysed the role of angiogenic factors (VEGF and its receptors, VEGFR-1 and VEGFR-2) in underlying mechanism of neovascularisation in PTC in compared with the commonest type of benign thyroid lesions; which is NH. VEGF is an important stimulator of angiogenesis. It has been implicated in the proliferation and spread of different malignant tumours. VEGF signals via two high-affinity tyrosine kinase receptors, VEGFR-1/Flt-1 and VEGFR-2/KDR, expressed mostly in endothelial cells (Vieira et al., 2005). VEGFR-1 and VEGFR-2 are expressed by most of the differentiated thyroid carcinoma. However, there is no correlation between the expression of VEGFR-2 in tumor tissue and the clinical response to lenvatinib among patients with anaplastic thyroid carcinoma (Yamazaki et al., 2018)

Different steps of tumour progression or invasion may involve different kinds of growth factors. This study expected to see the role of VEGF in invading vascular sprouts, providing an initial angiogenic signal and accompanying tumour growth. The process of neovascularisation is thought to be mediated by a tumour angiogenic factor, which is produced by the neoplastic cells. Numerous potential angiogenic factors have been characterised including vascular endothelial growth factor/vascular permeability factor (VEGF/VPF) (Katoh et al., 1999). VEGF may play a role in the regulation of development and function of thyroid epithelial cells and also thyroid tumour progression from a pre-vascular phase to a vascular phase. VEGF expression in tumour cells of thyroid cancer can induce neovascularisation (Gulobova et al., 2014).

In this study, findings were in accordance with other several studies (Jebreel et al., 2007; Katoh et al., 1999) in VEGF expression. However, this study hypothesised that there was a difference in VEGF and VEGF receptors (VEGFR) expression between both $\mathrm{NH}$ and PTC. Following our findings that were exhibiting overexpression of VEGF in thyroid disease including NH and PTC, Jebreel et al., (2007) found that all PTC (7 out of 7 cases) and 94\% (16) of multinodular goitre were immunopositive for VEGF. The intensity of VEGF staining showed stronger intensity in thyroid cells compared to normal thyroid cells which in accordance with findings by Soh et al., (1997). However, it was unable to demonstrate the statistical difference $(p=0.576)$ between NH and PTC in VEGF expression in this study.

In this study, there was a statistical difference in VEGFR-1 $(p=0.028)$ and VEGFR-2 $(p=0.003)$ expression between NH and PTC. The data showed that both VEGFR-1 and VEGFR-2 were more expressed in PTC.
These findings were similar with another study which also demonstrated higher expression of VEGFR-1 and VEGFR-2 in PTC (Vieira et al., 2005). On the other hand, it was observed that the expression of VEGFR-1 and VEGFR-2 showed minimal variation between the differing diseases. This finding was also seen in other research which suggested that the up-regulation of VEGF expression rather than its receptors are important in these diseases (Jebreel et al., 2007).

Similar to the findings of a study by Jebreel et al., (2007), VEGF was co-expressed with its receptors in both benign and malignant thyroid disease. In a study conducted among thyroid carcinomas of follicular origin, there was co-expression of VEGF and its receptors in $50 \%$ of PTC, $39 \%$ of follicular thyroid carcinomas and $12 \%$ of poorly differentiated thyroid carcinomas. That study found out the role of VEGF and VEGF receptor (VEGFR) pathways in thyroid tumorigenesis. By using human PTC-derived cell line (NPA'87), co-expression of VEGF and its receptors (VEGFR) in NPA'87 cells were demonstrated. The NPA'87 cells express both VEGF (three VEGF isoforms including VEGF-121, VEGF-165 and VEGF-189) and VEGF receptors (VEGFR-1/Flt and VEGFR-2/KDR). These findings were raising the possibility that VEGF may signal in an autocrine loop in these neoplasms, as observed previously for another type of cancers. The autocrine VEGF signalling plays an important role in thyroid carcinogenesis (Vieira et al., 2005).

In this study, co-expression of VEGF and VEGFR-1 in both $\mathrm{NH}(\mathrm{p}=0.016)$ and PTC $(\mathrm{p}=0.03)$ was more evident than the co-expression of VEGF and VEGFR-2. That finding was also seen in the previous study by Katoh et al., (1999), demonstrated that the intensity and distribution of the tumour cells overlying the neoplastic papillae of papillary carcinoma stained more intensely than the neoplastic cells forming follicles. In some cases of a follicular variant of PTC, the intensity of expression was high; however, there was focal distribution. In accordance with our findings, both tumour cells and the endothelial lining of blood vessels showed immunopositivity for VEGFR-1 and VEGFR-2 staining (Vieira et al., 2005). Focal endothelial VEGF staining was also noted in normal and neoplastic thyroid tissue (Katoh et al., 1999). These findings may propose the relationship between VEGF and its receptors (VEGFR) expression and the vascular architectures in thyroid neoplasia, especially in reference to morphogenesis of papillae of PTC. The adjacent isolated thyroid follicles were also stained distinctively with antibodies against human VEGF, VEGFR-1 and VEGFR-1.

The presence of extra-thyroidal extension and its implications are important in the surgical management of thyroid cancer to achieve complete tumour resection. Individuals presented with extra-thyroidal extension have a high incidence of local recurrence, which is commonly seen in older individuals or poorly differentiated histology. Incomplete resection of locally aggressive thyroid cancer has a high incidence of local recurrence with the mortality rate in the range of almost $50 \%$ (Shaha et al., 2006).

A study showed that the large tumour size in PTC is 
associated with lymph node metastasis and extrathyroid extension. They also demonstrated that there were differences between younger patients $(<55$ years $)$ and older groups ( $\geq 55$ years). Extrathyroid extension of primary tumours (Ex) was significant in older patients while lymph node metastasis $(\mathrm{N})$ was a relevant prognostic factor for local and distant recurrences and carcinoma death in younger women. In older age group, the male showed that lymph node metastasis had a prognostic impact similar to the extrathyroid extension of primary tumours (Ex) for local recurrence while extranodal carcinoma extension (LN-Ex) was the strongest prognostic factor for carcinoma death (Ito et al., 2011).

The VEGFR-1 expression is related to lymph node metastasis, and serum VEGF may be a marker of progression in the follow-up of patients with differentiated thyroid carcinoma (Karaca et al., 2011). The expressions of VEGF in PTC with lymph node metastasis were significantly higher than those without lymph node metastasis (Gong et al., 2012). LNM occurred more frequently in patients with high VEGF expression than in those with low VEGF expression (Huang et al., 2017). In this study, there was no relevant association between lymph node status with younger and older age groups $(\mathrm{p}=0.712)$ and gender $(\mathrm{p}=0.146)$. There was also no significant association found between extrathyroid extension with age groups ( $p>0.95)$ and gender $(\mathrm{p}>0.95)$.

VEGF and its receptors expression appear to be related to thyroid tumour behaviour that mediates endothelial cell proliferation and neovascularisation in thyroid tumours (Jebreel et al., 2007; Katoh et al., 1999). Many reports have correlated the increased expression of VEGF in thyroid carcinoma with a higher risk of recurrence and metastasis (Bunone et al., 1999; Jalali Nadoushan et al., 2012; Lennard et al., 2001). Shaha et al., (2006) showed that the elevated expression of VEGF mRNA in thyroid cancers is associated with high tumorigenic potential, and can be an important event in the transition from low to high-grade tumours.

All the three VEGFRs have distinct functions in separate tissue compartments. Thus, they might also converge functionally to promote pathological vessel formation in certain diseases. VEGFR-1 directs migration of monocytes or macrophages in tumour disease. VEGFR-2 causes overexpression and excess signal transduction in endothelial cells in diseases involving deregulated blood-vessel formation, such as tumours. VEGFR-3 (also known as Flt-4) is a $195-k D a$ high-affinity receptor for VEGF-C and VEGF-D. It causes overexpression and excess function in tumour lymphangiogenesis and lymph node metastasis. However, in general, all three VEGFRs are also crucial for vascular development and is expressed in certain normal vessels and tumour vessels (Cross et al., 2003).

Lymph node metastases of thyroid tumours showed increased VEGF expression with respect to a primary tumour. Bunone et al., (1999) found increased expression of VEGF-C in lymph node invasive thyroid tumours. Concomitant high expression of survivin and VEGF-C is closely associated with LNM status of PTC patients, which suggests their cooperation in the metastatic process (Selemetjev et al., 2018). However, VEGF-C is a thyroid marker of malignancy superior to VEGF-A in the differential diagnostics of thyroid lesions (Woliński et al., 2016). Chao et al., (2014) reported that VEGFR-3 is independently associated with cervical lymph node metastasis in papillary thyroid carcinoma.

Findings in this study were not in accordance to some previous studies. There was no significant association found between extrathyroid extension with expression of VEGF ( $p>0.95)$, VEGFR-1 $(p=0.352)$ and VEGFR-2 $(p=0.486)$. Furthermore, there was also no association between lymph node status with the expression of VEGF $(\mathrm{p}>0.95)$, VEGFR-1 $(\mathrm{p}=0.178)$ and VEGFR-2 $(\mathrm{p}>0.95)$. The interest of our study was VEGFR-1 and VEGFR-2 which were not highly potential for lymph node metastasis if compared to VEGFR-3.

Association of VEGF and VEGFR expression with prognostic factors had been demonstrated in several previous studies. This finding is useful to guide the clinicians for appropriate and optimum management of thyroid cancer patient, especially in an advanced stage or with iodine therapy resistant. In this study, we only managed to get the intraoperative and histopathologic findings of prognostic factors such as lymph node metastasis and extrathyroid extension. An assessment by acquired radiological images and reports for all cases may be helpful in the record for distant metastasis and also lymph node involvement in order to complete staging of each patient. However, the results were only limited based on the type of specimen that had been submitted for histopathological assessment.

In this study, there was no statistical difference in VEGF expression between NH and PTC. However, we found a statistical difference in VEGFR-1 and VEGFR-2 expression between NH and PTC. It was suggested that VEGFRs showed overexpression in PTC. There was no association between lymph node status and extrathyroid extension with age groups and gender and no association between lymph node status and extrathyroid extension with an expression of VEGF, VEGFR-1 and VEGFR-2. Co-expression of VEGF and VEGFR-1 was significant in both $\mathrm{NH}(\mathrm{p}=0.016)$ and PTC $(\mathrm{p}=0.03)$, meanwhile no significant relationship for VEGF and VEGFR-2 expression.

VEGF and VEGFR were highly expressed in thyroid disease. Nowadays VEGFR-targeted tyrosine kinase inhibitors have become available for the treatment of recurrent or metastatic thyroid cancer. However, careful consideration of the optimal timing of VEGFR-targeted TKI is needed to prevent distant metastasis (Tahara M., 2018). In a future study, pre-operative evaluation and intraoperative assessment of prognostic factors may be providing better results. Selection of additional antibodies such as VEGF-C and VEGFR-3 may also contributing to demonstrate the association of overexpression with malignancy and clinicopathological data.

In conclusion, VEGF, VEGFR-1 and VEGFR-2 showed overexpression in both NH and PTC. The expression of VEGFR-1 and VEGFR-2 were more significant in PTC with relevant co-expression of VEGF and VEGFR-1. Additional antibodies such as VEGF-C 
and VEGFR-3 may also contributing to demonstrate the association of overexpression with malignancy and clinicopathological data.

\section{Statement conflict of Interest}

No conflict of interest.

\section{Acknowledgements}

We would like to acknowledge Universiti Sains Malaysia for providing us with a short-term research grant (304/PPSP/61313054) to complete the study.

\section{References}

Abdel-Rahman O (2015). Targeting vascular endothelial growth factor (VEGF) pathway in iodine-refractory differentiated thyroid carcinoma (DTC): From bench to bedside. Crit Rev Oncol Hematol, 94, 45-54.

Bunone G, Vigneri P, Mariani L, et al (1999). Expression of angiogenesis stimulators and inhibitors in human thyroid tumours and correlation with clinical pathological features. Am J Pathol, 155, 1967-76.

Chao TT, Maa HC, Hsia TL, et al (2014). Vascular endothelial growth factor receptor-3 is independently associated with cervical lymph node metastasis in papillary thyroid carcinoma. Eur J Oncol, 19, 150-8.

Cross MJ, Dixelius J, Matsumoto T, Claesson-Welsh L (2003). VEGF-receptor signal transduction. Trends Biochem Sci, 28, 488-94.

Dvorak HF, Brown LF, Detmar M, Dvorak AM (1995). Vascular permeability factor/vascular endothelial growth factor, microvascular hyperpermeability, and angiogenesis. $\mathrm{Am} \mathrm{J}$ Pathol, 146, 1029-39.

Gandolfi PP, Frisina A, Raffa M, et al (2004). The incidence of thyroid carcinoma in multinodular goitre: retrospective analysis. Acta Biomed Ateneo Parmense, 75, 114-7.

Gong L, Chen P, Liu X, et al (2012). Expressions of D2-40, CK19, galectin-3, VEGF and EGFR in papillary thyroid carcinoma. Gland Surg, 1, 25-32.

Gulubova M, Ivanova K, Ananiev J, et al (2014). VEGF expression, microvessel density and dendritic cell decrease in thyroid cancer. Biotechnol Biotechnol Equip, 228, 508-17.

Huang XQ, He WS, Zhang HQ, Yang R, Huang T (2017). Relationship between expression of vascular endothelial growth factor and cervical lymph node metastasis in papillary thyroid cancer: A meta-analysis. J Huazhong Univ Sci Technolog Med Sci, 37, 661-6.

Ito Y, Kudo T, Takamura Y, et al (2011). Prognostic factors of papillary thyroid carcinoma vary according to sex and patient age. World J Surg, 35, 2684-90.

Jalali Nadoushan M, Siadati S, Amin A, Davat, A, Mirzapour M (2012). Study of correlation between vascular endothelial growth factor receptor and some prognostic factors in papillary carcinoma of thyroid gland. J Basic Clin Physiol Pharmacol, 1, 1-7.

Jebreel A, England J, Bedford K, et al (2007). Vascular endothelial growth factor (VEGF), VEGF receptors expression and microvascular density in benign and malignant thyroid diseases. Int J Exp Pathol, 88, 271-7.

Johnson KE, Wilgus TA (2012). Multiple roles for VEGF in non-melanoma skin cancer: Angiogenesis and Beyond. J Skin Cancer, 2012, 483439.

Karaca Z, Tanriverdi F, Unluhizarci K, et al (2011). VEGFR1 expression is related to lymph node metastasis and serum VEGF may be a marker of progression in the follow-up of patients with differentiated thyroid carcinoma. Eur $J$ Endocrinol, 164, 277-84.

Katoh R, Miyagi E, Kawaoi A, et al (1999). Expression of vascular endothelial growth factor (VEGF) in human thyroid neoplasms. Hum Pathol, 130, 891-7.

Klein M, Vignaud JM, Hennequin V, et al (2001). Increased expression of the vascular endothelial growth factor is a pejorative prognosis marker in papillary thyroid carcinoma. $J$ Clin Endocrinol Metab, 86, 656-8.

Lennard CM, Patel A, Wilson J, et al (2001). Intensity of vascular endothelial growth factor expression is associated with increased risk of recurrence and decreased disease-free survival in papillary thyroid cancer. Surgery, 129, 552-8.

Othman NH, Omar E, Naing NN (2009). Spectrum of thyroid lesions in Hospital Universiti Sains Malaysia over 11 years and a review of thyroid cancers in Malaysia. Asian Pac J Cancer Prev, 10, 87-90.

Parameswaran R, Brooks S, Sadler GP (2010). Molecular pathogenesis of follicular cell derived thyroid cancers. Int J Surg, 8, 186-93.

Peng XG, Chen ZF, Zhang KJ, et al (2015). VEGF Trapon inhibits tumour growth in papillary thyroid carcinoma. Eur Rev Med Pharmacol Sci, 19, 235-40.

Sachmechi I, Miller E, Varatharajah R, et al (2000). Thyroid carcinoma in single cold nodules and in cold nodules of multinodular goitres. Endocr Pract, 6, 5-7.

Selemetjev S, Savin S, Paunovic I, et al (2018). Concomitant high expression of survivin and vascular endothelial growth factor-C is strongly associated with metastatic status of lymph nodes in papillary thyroid carcinoma. J Can Res Ther, 14, 114-9.

Shaha A (2006). Treatment of thyroid cancer based on risk groups. J Surg Oncol, 94, 683-91.

Soh EY, Duh QY, Sobhi SA, et al (1997). Vascular endothelial growth factor expression is higher in differentiated thyroid cancer than in normal or benign thyroid. J Clin Endocrinol Metab, 82, 3741-7.

Tahara M (2018). Management of recurrent or metastatic thyroid cancer. ESMO Open, 3, e000359.

Vieira JM, Santos SCR, Espadinha C, et al (2005). Expression of vascular endothelial growth factor (VEGF) and its receptors in thyroid carcinomas of follicular origin: a potential autocrine loop. Eur J Endocrinol, 153, 701-9.

Woliński K, Stangierski A, Szczepanek-Parulska E, et al (2016). VEGF-C is a thyroid marker of malignancy superior to VEGF-A in the differential diagnostics of thyroid lesions. PLoS One, 11, e0150124.

Yamazaki H, Yokose T, Hayashi H, et al (2018). Expression of vascular endothelial growth factor receptor 2 and clinical response to lenvatinib in patients with anaplastic thyroid cancer. Cancer Chemother Pharmacol, 82, 649-54.

Younes MN, Yazici YD, Kim S, Jasser SA, et al (2006). Dual epidermal growth factor receptor and vascular endothelial growth factor receptor inhibition with nvp-aee78 for the treatment of aggressive follicular thyroid cancer. Clin Cancer Res, 12, 3425-34.

Zhou ZH, Cui XN, Xing HG, et al (2012). Changes and prognostic value of serum vascular endothelial growth factor in patients with differentiated thyroid cancer. Med Princ Pract, 22, 24-8.

This work is licensed under a Creative Commons AttributionNon Commercial 4.0 International License. 